\title{
EXTRAPOLATION OF PERIODICALLY CORRELATED STOCHASTIC PROCESSES OBSERVED WITH NOISE
}

UDC 519.21

\section{I. DUBOVETS'KA AND M. P. MOKLYACHUK}

\begin{abstract}
We consider the problem of the optimal linear estimation of the functional

$$
A \zeta=\int_{0}^{\infty} a(t) \zeta(t) d t
$$

depending on unknown values of a periodically correlated stochastic process $\zeta(t)$. An estimator is constructed from observations of the process $\zeta(t)+\theta(t)$ for $t<0$, where $\theta(t)$ is a periodically correlated process being uncorrelated with $\zeta(t)$. Formulas for calculating the spectral characteristic and the mean square error of the optimal linear estimator of the functional are proposed in the case where spectral densities are known. In the case where spectral densities are not known but a set of admissible spectral densities is specified, formulas that determine the least favorable spectral density and the minimax (robust) spectral characteristics of optimal estimators of the above functional are proposed.
\end{abstract}

\section{INTRODUCTION}

The methods for the investigation of problems of estimation of unknown values of stationary stochastic processes (extrapolation, interpolation, and filtration problems) have been developed by Kolmogorov [8, Wiener [18, Yaglom [16, 17, and Rozanov [15]. These methods are based on the assumption that the spectral densities are known. If the corresponding spectral densities are not known but a set of admissible spectral densities is specified, then one can follow the minimax method when solving estimation problems. The minimax method reduces to the minimization of the error for all densities belonging to a specified set simultaneously. Grenander [2] was the first to apply the minimax approach to the extrapolation problem for stationary stochastic processes. A survey of publications related to minimax (robust) methods is presented by Kassam and Poor [7]. Moklyachuk [12] and Moklyachuk and Masyutka [13] study the problems of extrapolation, interpolation, and filtration for stationary stochastic processes and sequences (also see the book by Kurkin, Korobochkin, and Shatalov [9]).

Studies of periodically correlated processes were initiated by Gladyshev [1, who investigated the properties of covariance functions and spectral representations of periodically correlated processes. A relationship between periodically correlated and infinite dimensional stationary processes is considered by Makagon [10, 11]. The minimax problems of the optimal estimation of linear functionals are studied by Dubovets'ka, Masyutka, and Moklyachuk [3]-[5].

2010 Mathematics Subject Classification. Primary 60G10, 60G25, 60G35; Secondary 62M20, 93E10, $93 \mathrm{E} 11$.

Key words and phrases. Periodically correlated processes, minimax estimator, mean square error, least favorable spectral density. 
In the current paper, we study the problem of the mean square linear optimal estimation of the functional

$$
A \zeta=\int_{0}^{\infty} a(t) \zeta(t) d t
$$

constructed from the values of a mean square continuous periodically correlated stochastic process $\zeta(t)$. The above functional is estimated from observations of the process $\zeta(t)+\theta(t)$ for $t<0$, where $\theta(t)$ is a mean square continuous periodically correlated process being uncorrelated with $\zeta(t)$. We find formulas for calculating the spectral characteristic and mean square error of the optimal estimator of the functional $A \zeta$ in the case where the spectral densities of the stationary sequences $\left\{\zeta_{j}, j \in \mathbb{Z}\right\}$ and $\left\{\theta_{j}, j \in \mathbb{Z}\right\}$ are known. If the complete information about spectral densities is not available but a set of admissible spectral densities is specified, then we follow the minimax approach for solving the estimation problems. We find the least favorable spectral densities and minimax spectral characteristic of the optimal estimator of the functional $A \zeta$ for some classes of admissible spectral densities.

\section{Periodically correlated processes and CORResponding Vector VAlued STATIONARY SEQUENCES}

Definition 2.1 ([1]). A mean square continuous stochastic process

$$
\zeta: \mathbb{R} \rightarrow H=L_{2}(\Omega, \mathcal{F}, \mathrm{P}), \quad \mathrm{E} \zeta(t)=0,
$$

is called periodically correlated with period $T$ if its correlation function $K(t+u, u)=$ $\mathrm{E} \zeta(t+u) \overline{\zeta(u)}$ is such that

$$
K(t+u, u)=\mathrm{E} \zeta(t+u+T) \overline{\zeta(u+T)}=K(t+u+T, u+T)
$$

for all $t, u \in \mathbb{R}$.

Let $\zeta(t), t \in \mathbb{R}$, and $\theta(t), t \in \mathbb{R}$, be uncorrelated periodically correlated stochastic processes. We construct two sequences of random functions:

$$
\begin{aligned}
& \left\{\zeta_{j}(u)=\zeta(u+j T), u \in[0, T), j \in \mathbb{Z}\right\}, \\
& \left\{\theta_{j}(u)=\theta(u+j T), u \in[0, T), j \in \mathbb{Z}\right\} .
\end{aligned}
$$

Each of the sequences (11) or (2) generates an $L_{2}([0, T) ; H)$ valued stationary sequence $\left\{\zeta_{j}, j \in \mathbb{Z}\right\}$ or $\left\{\theta_{j}, j \in \mathbb{Z}\right\}$, respectively, whose correlation functions are given by

$$
\begin{aligned}
B_{\zeta}(l, j) & =\left\langle\zeta_{l}, \zeta_{j}\right\rangle_{H}=\int_{0}^{T} \mathrm{E}[\zeta(u+l T) \overline{\zeta(u+j T)}] d u \\
& =\int_{0}^{T} K_{\zeta}(u+(l-j) T, u) d u=B_{\zeta}(l-j), \\
B_{\theta}(l, j) & =\left\langle\theta_{l}, \theta_{j}\right\rangle_{H}=\int_{0}^{T} \mathrm{E}[\theta(u+l T) \overline{\theta(u+j T)}] d u \\
& =\int_{0}^{T} K_{\theta}(u+(l-j) T, u) d u=B_{\theta}(l-j),
\end{aligned}
$$

where $K_{\zeta}(t, s)=\mathrm{E} \zeta(t) \overline{\zeta(s)}$ and $K_{\theta}(t, s)=\mathrm{E} \theta(t) \overline{\theta(s)}$ are the correlation functions of the periodically correlated processes $\zeta(t)$ and $\theta(t)$, respectively.

If

$$
\left\{\widetilde{e}_{k}=\frac{1}{\sqrt{T}} \exp \left\{2 \pi i\left\{(-1)^{k}\left[\frac{k}{2}\right]\right\} u / T\right\}, k=1,2,3, \ldots\right\}, \quad\left\langle\widetilde{e}_{j}, \widetilde{e}_{k}\right\rangle=\delta_{k j},
$$


is an orthonormal basis in $L_{2}([0, T) ; \mathbb{R})$, then the stationary sequences $\left\{\zeta_{j}, j \in \mathbb{Z}\right\}$ and $\left\{\theta_{j}, j \in \mathbb{Z}\right\}$ admit the representations

$$
\begin{gathered}
\zeta_{j}=\sum_{k=1}^{\infty} \zeta_{k j} \widetilde{e}_{k} \\
\zeta_{k j}=\left\langle\zeta_{j}, \widetilde{e}_{k}\right\rangle=\frac{1}{\sqrt{T}} \int_{0}^{T} \zeta_{j}(v) \exp \left\{-2 \pi i\left\{(-1)^{k}\left[\frac{k}{2}\right]\right\} v / T\right\} d v
\end{gathered}
$$

and

$$
\theta_{j}=\sum_{k=1}^{\infty} \theta_{k j} \widetilde{e}_{k}
$$

$$
\theta_{k j}=\left\langle\theta_{j}, \widetilde{e}_{k}\right\rangle=\frac{1}{\sqrt{T}} \int_{0}^{T} \theta_{j}(v) \exp \left\{-2 \pi i\left\{(-1)^{k}\left[\frac{k}{2}\right]\right\} v / T\right\} d v
$$

The components $\zeta_{k j}, k=1,2, \ldots$, and $\theta_{k j}, k=1,2, \ldots$, of the stationary sequences $\left\{\zeta_{j}, j \in \mathbb{Z}\right\}$ and $\left\{\theta_{j}, j \in \mathbb{Z}\right\}$ are such that

$$
\begin{aligned}
& \mathrm{E} \zeta_{k j}=0, \quad\left\|\zeta_{j}\right\|_{H}^{2}=\sum_{k=1}^{\infty} \mathrm{E}\left|\zeta_{k j}\right|^{2}=P_{\zeta}<\infty, \quad \mathrm{E} \zeta_{k l} \overline{\zeta_{n j}}=\left\langle R_{\zeta}(l-j) e_{k}, e_{n}\right\rangle, \\
& \mathrm{E} \theta_{k j}=0, \quad\left\|\theta_{j}\right\|_{H}^{2}=\sum_{k=1}^{\infty} \mathrm{E}\left|\theta_{k j}\right|^{2}=P_{\theta}<\infty, \quad \mathrm{E} \theta_{k l} \overline{\theta_{n j}}=\left\langle R_{\theta}(l-j) e_{k}, e_{n}\right\rangle
\end{aligned}
$$

(see [6]), where $\left\{e_{k}, k=1,2, \ldots\right\}$ is a basis in the space $\ell_{2}$. The correlation functions $R_{\zeta}(j)$ and $R_{\theta}(j)$ of the stationary sequences $\left\{\zeta_{j}, j \in \mathbb{Z}\right\}$ and $\left\{\theta_{j}, j \in \mathbb{Z}\right\}$ are operator valued functions in $\ell_{2}$. The correlation operators $R_{\zeta}(0)=R_{\zeta}$ and $R_{\theta}(0)=R_{\theta}$ are kernel operators

$$
\begin{aligned}
& \sum_{k=1}^{\infty}\left\langle R_{\zeta} e_{k}, e_{k}\right\rangle=\left\|\zeta_{j}\right\|_{H}^{2}=P_{\zeta}, \\
& \sum_{k=1}^{\infty}\left\langle R_{\theta} e_{k}, e_{k}\right\rangle=\left\|\theta_{j}\right\|_{H}^{2}=P_{\theta} .
\end{aligned}
$$

The stationary sequences $\left\{\zeta_{j}, j \in \mathbb{Z}\right\}$ and $\left\{\theta_{j}, j \in \mathbb{Z}\right\}$ have spectral densities $f(\lambda)=$ $\left\{f_{k n}(\lambda)\right\}_{k, n=1}^{\infty}$ and $g(\lambda)=\left\{g_{k n}(\lambda)\right\}_{k, n=1}^{\infty}$ that are positive operator valued functions in $\ell_{2}$ of the argument $\lambda \in[-\pi, \pi)$ if their correlation functions $R_{\zeta}(j)$ and $R_{\theta}(j)$ can be written as follows:

$$
\begin{gathered}
\left\langle R_{\zeta}(j) e_{k}, e_{n}\right\rangle=\frac{1}{2 \pi} \int_{-\pi}^{\pi} e^{i j \lambda}\left\langle f(\lambda) e_{k}, e_{n}\right\rangle d \lambda \\
\left\langle R_{\theta}(j) e_{k}, e_{n}\right\rangle=\frac{1}{2 \pi} \int_{-\pi}^{\pi} e^{i j \lambda}\left\langle g(\lambda) e_{k}, e_{n}\right\rangle d \lambda, \quad k, n \geq 1 .
\end{gathered}
$$

For almost all $\lambda \in[-\pi, \pi)$, the spectral densities $f(\lambda)$ and $g(\lambda)$ are kernel operators with integrable kernel norms

$$
\begin{aligned}
& \sum_{k=1}^{\infty} \frac{1}{2 \pi} \int_{-\pi}^{\pi}\left\langle f(\lambda) e_{k}, e_{k}\right\rangle d \lambda=\sum_{k=1}^{\infty}\left\langle R_{\zeta} e_{k}, e_{k}\right\rangle=\left\|\zeta_{j}\right\|_{H}^{2}=P_{\zeta} \\
& \sum_{k=1}^{\infty} \frac{1}{2 \pi} \int_{-\pi}^{\pi}\left\langle g(\lambda) e_{k}, e_{k}\right\rangle d \lambda=\sum_{k=1}^{\infty}\left\langle R_{\theta} e_{k}, e_{k}\right\rangle=\left\|\theta_{j}\right\|_{H}^{2}=P_{\theta} .
\end{aligned}
$$




\section{Classical method of linear extrapolation OF PERIODICALLY CORRELATED PROCESSES}

We study the problem of the mean square optimal linear estimation of the functional

$$
A \zeta=\int_{0}^{\infty} a(t) \zeta(t) d t
$$

constructed from the values of a mean square continuous periodically correlated stochastic process $\zeta(t)$. The functional $A$ is estimated from observations of the process $\zeta(t)+\theta(t)$ for $t<0$, where $\theta(t)$ is an uncorrelated with $\zeta(t)$ mean square continuous periodically correlated process. The function $a(t), t \in \mathbb{R}_{+}$, is such that

$$
\int_{0}^{\infty}|a(t)| d t<\infty
$$

We rewrite the functional $A \zeta$ as follows:

$$
\begin{gathered}
A \zeta=\int_{0}^{\infty} a(t) \zeta(t) d t=\sum_{j=0}^{\infty} \int_{0}^{T} a_{j}(u) \zeta_{j}(u) d u \\
a(u+j T)=a_{j}(u), \quad \zeta(u+j T)=\zeta_{j}(u), \quad u \in[0, T) .
\end{gathered}
$$

Recalling decomposition (3) of the stationary sequence $\left\{\zeta_{j}, j \in \mathbb{Z}\right\}$, the functional $A \zeta$ can be rewritten as

$$
\begin{aligned}
A \zeta= & \sum_{j=0}^{\infty} \int_{0}^{T} a_{j}(u) \zeta_{j}(u) d u \\
= & \sum_{j=0}^{\infty} \frac{1}{T} \int_{0}^{T}\left(\sum_{k=1}^{\infty} a_{k j} \exp \left\{2 \pi i\left\{(-1)^{k}\left[\frac{k}{2}\right]\right\} u / T\right\}\right) \\
& \times\left(\sum_{n=1}^{\infty} \zeta_{n j} \exp \left\{2 \pi i\left\{(-1)^{n}\left[\frac{n}{2}\right]\right\} u / T\right\}\right) d u \\
= & \sum_{j=0}^{\infty} \sum_{k=1}^{\infty} \sum_{n=1}^{\infty} a_{k j} \zeta_{n j} \frac{1}{T} \int_{0}^{T} \exp \left\{2 \pi i\left\{(-1)^{k}\left[\frac{k}{2}\right]+(-1)^{n}\left[\frac{n}{2}\right]\right\} u / T\right\} d u \\
= & \sum_{j=0}^{\infty} \sum_{k=1}^{\infty} a_{k j} \zeta_{k j}=\sum_{j=0}^{\infty} \vec{a}_{j}^{\top} \vec{\zeta}_{j},
\end{aligned}
$$

since solutions of the equation

$$
(-1)^{k}\left[\frac{k}{2}\right]+(-1)^{n}\left[\frac{n}{2}\right]=0
$$

with respect to the pair of unknowns $(k, n)$ are $(1,1),(2 l+1,2 l)$, and $(2 l, 2 l+1)$ for $l=2,3, \ldots$, where

$$
\begin{gathered}
\vec{\zeta}_{j}=\left(\zeta_{k j}, k=1,2, \ldots\right)^{\top}, \\
\vec{a}_{j}=\left(a_{k j}, k=1,2, \ldots\right)^{\top}=\left(a_{1 j}, a_{3 j}, a_{2 j}, \ldots, a_{2 k+1, j}, a_{2 k, j}, \ldots\right)^{\top}, \\
a_{k j}=\left\langle a_{j}, \widetilde{e}_{k}\right\rangle=\frac{1}{\sqrt{T}} \int_{0}^{T} a_{j}(v) \exp \left\{-2 \pi i\left\{(-1)^{k}\left[\frac{k}{2}\right]\right\} v / T\right\} d v .
\end{gathered}
$$

Assume that the coefficients $\left\{\vec{a}_{j}, j=0,1, \ldots\right\}$ are such that

$$
\sum_{j=0}^{\infty}\left\|\vec{a}_{j}\right\|<\infty, \quad \sum_{j=0}^{\infty}(j+1)\left\|\vec{a}_{j}\right\|^{2}<\infty, \quad\left\|\vec{a}_{j}\right\|^{2}=\sum_{k=1}^{\infty}\left|a_{k j}\right|^{2} .
$$


According to the first condition in (6), the second moment of the functional $A \zeta$ is finite. The second condition in (6) implies the compactness of the operators to be defined below.

Let the spectral densities $f(\lambda)$ and $g(\lambda)$ of the sequences $\left\{\zeta_{j}, j \in \mathbb{Z}\right\}$ and $\left\{\theta_{j}, j \in \mathbb{Z}\right\}$, respectively, satisfy the following minimality condition:

$$
\int_{-\pi}^{\pi} \operatorname{Tr}\left[(f(\lambda)+g(\lambda))^{-1}\right] d \lambda<\infty .
$$

Note that condition (7) is necessary and sufficient for the error free extrapolation of unknown values of the sequence $\left\{\zeta_{j}+\theta_{j}, j \in \mathbb{Z}\right\}$ to be impossible (see [15, 12]).

Denote by $L_{2}(f)$ the Hilbert space of vector complex valued functions

$$
b(\lambda)=\left\{b_{k}(\lambda)\right\}_{k=1}^{\infty}
$$

that are square integrable with respect to the Lebesgue measure and that have the density $f(\lambda)$ :

$$
\int_{-\pi}^{\pi} b^{\top}(\lambda) f(\lambda) \overline{b(\lambda)} d \lambda=\int_{-\pi}^{\pi} \sum_{k, n=1}^{\infty} b_{k}(\lambda) \overline{b_{n}(\lambda)} f_{k n}(\lambda) d \lambda<\infty .
$$

We denote by $L_{2}^{-}(f)$ the subspace of $L_{2}(f)$ generated by the functions

$$
e^{i j \lambda} \delta_{k}, \quad k=1,2, \ldots, j<0,
$$

where

$$
\delta_{k}=\left\{\delta_{k n}\right\}_{n=1}^{\infty}
$$

and $\delta_{k k}=1, \delta_{k n}=0$ for $k \neq n$.

Any linear estimator $\hat{A} \zeta$ of the functional $A \zeta$ constructed from the observations of the sequence $\left\{\zeta_{j}+\theta_{j}\right\}, j<0$, can be written as follows:

$$
\hat{A} \zeta=\int_{-\pi}^{\pi} h^{\top}\left(e^{i \lambda}\right)\left(Z^{\zeta}(d \lambda)+Z^{\theta}(d \lambda)\right)=\int_{-\pi}^{\pi} \sum_{k=1}^{\infty} h_{k}\left(e^{i \lambda}\right)\left(Z_{k}^{\zeta}(d \lambda)+Z_{k}^{\theta}(d \lambda)\right),
$$

where $Z^{\zeta}(\Delta)=\left\{Z_{k}^{\zeta}(\Delta)\right\}_{k=1}^{\infty}$ and $Z^{\theta}(\Delta)=\left\{Z_{k}^{\theta}(\Delta)\right\}_{k=1}^{\infty}$ are orthogonal random measures of the sequences $\left\{\zeta_{j}\right\}$ and $\left\{\theta_{j}\right\}$, respectively, and where $h\left(e^{i \lambda}\right)=\left\{h_{k}\left(e^{i \lambda}\right)\right\}_{k=1}^{\infty}$ is the spectral characteristic of the estimator $\hat{A} \zeta$. Note also that $h\left(e^{i \lambda}\right) \in L_{2}^{-}(f+g)$.

The mean square error $\Delta(h ; f, g)$ of the estimator $\hat{A} \zeta$ is calculated from the following formula:

$$
\begin{aligned}
& \Delta(h ; f, g)=\mathrm{E}|A \zeta-\hat{A} \zeta|^{2} \\
&=\frac{1}{2 \pi} \int_{-\pi}^{\pi}\left(\left[A\left(e^{i \lambda}\right)-h\left(e^{i \lambda}\right)\right]^{\top} f(\lambda) \overline{\left[A\left(e^{i \lambda}\right)-h\left(e^{i \lambda}\right)\right]}\right.\left.+h^{\top}\left(e^{i \lambda}\right) g(\lambda) \overline{h\left(e^{i \lambda}\right)}\right) d \lambda \\
& A\left(e^{i \lambda}\right)=\sum_{j=0}^{\infty} \vec{a}_{j} e^{i j \lambda}
\end{aligned}
$$

The spectral characteristic $h(f, g)$ of the optimal linear estimator $\hat{A} \zeta$ minimizes the mean square error

$$
\Delta(f, g)=\Delta(h(f, g) ; f, g)=\min _{h \in L_{2}^{-}(f+g)} \Delta(h ; f, g)=\min _{\hat{A} \zeta} \mathrm{E}|A \zeta-\hat{A} \zeta|^{2} .
$$

The optimal linear error $\hat{A} \zeta$ is a solution of the optimization problem (10). 
When using the classical Kolmogorov method of projections [8] we write the following two conditions that determine $\hat{A} \zeta$ as a projection onto the subspace $H^{-}(\zeta+\theta)$ of the space $H$ generated by the random variables $\zeta_{j}+\theta_{j}, j<0$ :

1) $\hat{A} \zeta \in H^{-}(\zeta+\theta)$,

2) $(A \zeta-\hat{A} \zeta) \perp H^{-}(\zeta+\theta)$.

Condition 2) holds if

$$
\begin{gathered}
\mathrm{E}(A \zeta-\hat{A} \zeta)\left(\overline{\zeta_{j}+\theta_{j}}\right)=\frac{1}{2 \pi} \int_{-\pi}^{\pi}\left[\left(A\left(e^{i \lambda}\right)-h\left(e^{i \lambda}\right)\right)^{\top} f(\lambda)-h^{\top}\left(e^{i \lambda}\right) g(\lambda)\right] e^{-i j \lambda} d \lambda \\
=\frac{1}{2 \pi} \int_{-\pi}^{\pi}\left[A^{\top}\left(e^{i \lambda}\right) f(\lambda)-h^{\top}\left(e^{i \lambda}\right)(f(\lambda)+g(\lambda))\right] e^{-i j \lambda} d \lambda=0, \\
j=-1,-2, \ldots .
\end{gathered}
$$

The latter equality implies that

$$
A^{\top}\left(e^{i \lambda}\right) f(\lambda)-h^{\top}\left(e^{i \lambda}\right)(f(\lambda)+g(\lambda))=\sum_{j=0}^{\infty} \vec{c}_{j}^{\top} e^{i j \lambda}=C^{\top}\left(e^{i \lambda}\right),
$$

where $\vec{c}_{j}, j=0,1, \ldots$, are unknown coefficients. Thus the spectral characteristic $h(f, g)$ of the optimal estimator of the functional $A \zeta$ can be written as follows:

$$
\begin{aligned}
h^{\top}(f, g) & =\left(A^{\top}\left(e^{i \lambda}\right) f(\lambda)-C^{\top}\left(e^{i \lambda}\right)\right)[f(\lambda)+g(\lambda)]^{-1} \\
& =A^{\top}\left(e^{i \lambda}\right)-\left(A^{\top}\left(e^{i \lambda}\right) g(\lambda)+C^{\top}\left(e^{i \lambda}\right)\right)[f(\lambda)+g(\lambda)]^{-1} .
\end{aligned}
$$

Condition 1) above is equivalent to the equalities

$$
\int_{-\pi}^{\pi} h(f, g) e^{-i l \lambda} d \lambda=0, \quad l=0,1, \ldots
$$

that is,

$$
\begin{aligned}
\sum_{j=0}^{\infty} & \frac{1}{2 \pi} \int_{-\pi}^{\pi}\left[f(\lambda)(f(\lambda)+g(\lambda))^{-1}\right]^{\top} e^{i(j-l) \lambda} d \lambda \vec{a}_{j} \\
& =\sum_{j=0}^{\infty} \frac{1}{2 \pi} \int_{-\pi}^{\pi}\left[(f(\lambda)+g(\lambda))^{-1}\right]^{\top} e^{i(j-l) \lambda} d \lambda \vec{c}_{j}, \quad l=0,1, \ldots
\end{aligned}
$$

Introducing the matrices $\mathbf{B}=\{B(l, j)\}_{l, j=0}^{\infty}$ and $\mathbf{D}=\{D(l, j)\}_{l, j=0}^{\infty}$ constituted by the entries

$$
\begin{gathered}
B(l, j)=\frac{1}{2 \pi} \int_{-\pi}^{\pi}\left[(f(\lambda)+g(\lambda))^{-1}\right]^{\top} e^{i(j-l) \lambda} d \lambda, \\
D(l, j)=\frac{1}{2 \pi} \int_{-\pi}^{\pi}\left[f(\lambda)(f(\lambda)+g(\lambda))^{-1}\right]^{\top} e^{i(j-l) \lambda} d \lambda
\end{gathered}
$$

and vectors $\mathbf{a}=\left\{\vec{a}_{j}\right\}_{j=0}^{\infty}$ and $\mathbf{c}=\left\{\vec{c}_{j}\right\}_{j=0}^{\infty}$, the system of equations (12) can be written in the matrix form

$$
\mathbf{D a}=\mathbf{B c},
$$

whence we conclude that unknown coefficients $\vec{c}_{j}, j=0,1, \ldots$, are determined from the equation

$$
\mathbf{c}=\mathbf{B}^{-1} \mathbf{D a}
$$


In view of equality (9), the mean square error of the optimal estimator $\hat{A} \zeta$ is given by

$$
\begin{aligned}
\Delta(f, g)= & \Delta(h(f, g) ; f, g) \\
= & \frac{1}{2 \pi} \int_{-\pi}^{\pi}\left[A^{\top}\left(e^{i \lambda}\right) g(\lambda)+C^{\top}\left(e^{i \lambda}\right)\right][f(\lambda)+g(\lambda)]^{-1} \\
& \times f(\lambda)[f(\lambda)+g(\lambda)]^{-1}\left[A^{\top}\left(e^{i \lambda}\right) g(\lambda)+C^{\top}\left(e^{i \lambda}\right)\right]^{*} d \lambda \\
& +\frac{1}{2 \pi} \int_{-\pi}^{\pi}\left[A^{\top}\left(e^{i \lambda}\right) f(\lambda)-C^{\top}\left(e^{i \lambda}\right)\right][f(\lambda)+g(\lambda)]^{-1} \\
& \times g(\lambda)[f(\lambda)+g(\lambda)]^{-1}\left[A^{\top}\left(e^{i \lambda}\right) f(\lambda)-C^{\top}\left(e^{i \lambda}\right)\right]^{*} d \lambda .
\end{aligned}
$$

Using the following properties of spectral densities,

$$
\begin{gathered}
f(\lambda)=f^{*}(\lambda), \quad g(\lambda)=g^{*}(\lambda), \\
f(\lambda)[f(\lambda)+g(\lambda)]^{-1} g(\lambda)=g(\lambda)[f(\lambda)+g(\lambda)]^{-1} f(\lambda),
\end{gathered}
$$

the formula for calculating the mean square error $\Delta(f, g)$ can be rewritten as

$$
\begin{aligned}
\Delta(f, g)= & \sum_{j=0}^{\infty} \vec{a}_{j}^{\top} \overline{\sum_{l=0}^{\infty} \frac{1}{2 \pi} \int_{-\pi}^{\pi}\left[f(\lambda)(f(\lambda)+g(\lambda))^{-1} g(\lambda)\right]^{\top} e^{i(l-j) \lambda} d \lambda \vec{a}_{l}} \\
& +\sum_{j=0}^{\infty} \vec{c}_{j}^{\top} \overline{\sum_{l=0}^{\infty} \frac{1}{2 \pi} \int_{-\pi}^{\pi}\left[(f(\lambda)+g(\lambda))^{-1}\right]^{\top} e^{i(l-j) \lambda} d \lambda \vec{c}_{l}} \\
= & \langle\mathbf{a}, \mathbf{R a}\rangle+\langle\mathbf{c}, \mathbf{B} \mathbf{c}\rangle,
\end{aligned}
$$

where $\langle a, b\rangle$ means the scalar product in the space $\ell_{2}$ and where the matrix $\mathbf{R}=$ $\{R(j, l)\}_{j, l=0}^{\infty}$ is constructed from the entries

$$
R(j, l)=\frac{1}{2 \pi} \int_{-\pi}^{\pi}\left[f(\lambda)(f(\lambda)+g(\lambda))^{-1} g(\lambda)\right]^{\top} e^{i(l-j) \lambda} d \lambda, \quad l, j=0,1, \ldots .
$$

Combining all the above results we get the following assertion.

Theorem 3.1. Let $\{\zeta(t), t \in \mathbb{R}\}$ and $\{\theta(t), t \in \mathbb{R}\}$ be mutually uncorrelated periodically correlated stochastic processes such that both stationary sequences $\left\{\zeta_{j}, j \in \mathbb{Z}\right\}$ and $\left\{\theta_{j}, j \in \mathbb{Z}\right\}$ defined by equalities (11) and (2), respectively, possess the spectral densities $f(\lambda)$ and $g(\lambda)$ that satisfy the minimality condition (77).

Further assume that the coefficients $\left\{\vec{a}_{j}, j=0,1, \ldots\right\}$ defining the functional $A \zeta$ satisfy conditions (6).

Then the spectral characteristic $h(f, g)$ and mean square error $\Delta(f, g)$ of the optimal linear estimator of the functional $A \zeta$ constructed from the observations of the process $\zeta(t)+\theta(t), t<0$, are calculated by equalities (11) and (13), respectively. The optimal estimator $\hat{A} \zeta$ of the functional $A \zeta$ is calculated by formula (8).

Theorem 3.1 implies the following result for the extrapolation problem of the functional $A \zeta$ without noise.

Corollary 3.1. Let $\{\zeta(t), t \in \mathbb{R}\}$ be a periodically correlated stochastic process such that the stationary sequence $\left\{\zeta_{j}, j \in \mathbb{Z}\right\}$ defined by relations (1) possesses the spectral density $f(\lambda)$ that satisfies the following minimality condition:

$$
\int_{-\pi}^{\pi} \operatorname{Tr}\left[(f(\lambda))^{-1}\right] d \lambda<\infty .
$$

Let the coefficients $\left\{\vec{a}_{j}, j=0,1, \ldots\right\}$ defining the functional A $\zeta$ satisfy condition (6). Then the spectral characteristic $h(f)$ and mean square error $\Delta(f)$ of the optimal linear 
estimator of the functional $A \zeta$ constructed from observations of the process $\zeta(t), t<0$, are calculated as follows:

$$
\begin{gathered}
h^{\top}(f)=A^{\top}\left(e^{i \lambda}\right)-C^{\top}\left(e^{i \lambda}\right)[f(\lambda)]^{-1}, \\
\Delta(f)=\langle\mathbf{c}, \mathbf{a}\rangle,
\end{gathered}
$$

where $\mathbf{c}=\left\{\vec{c}_{j}\right\}_{j=0}^{\infty}=\mathbf{B}^{-1} \mathbf{a}$ and where the matrix $\mathbf{B}=\{B(l, j)\}_{l, j=0}^{\infty}$ is constructed from the entries

$$
B(l, j)=\frac{1}{2 \pi} \int_{-\pi}^{\pi}\left[(f(\lambda))^{-1}\right]^{\top} e^{i(j-l) \lambda} d \lambda, \quad l, j=0,1, \ldots
$$

The optimal estimator $\hat{A} \zeta$ of the functional $A \zeta$ is given by

$$
\hat{A} \zeta=\int_{-\pi}^{\pi} h^{\top}\left(e^{i \lambda}\right) Z^{\zeta}(d \lambda)=\int_{-\pi}^{\pi} \sum_{k=1}^{\infty} h_{k}\left(e^{i \lambda}\right) Z_{k}^{\zeta}(d \lambda) .
$$

Remark 3.1. Kolmogorov [8] used another method for solving the problem of interpolation of a stationary sequence (that is, for finding the spectral characteristic and mean square error of the optimal linear estimator for a missing observation in the sequence). Kolmogorov's method is based on a treatment of the Fourier coefficients of the function $1 / f$. Theorem 3.1 shows that the Fourier coefficients of functions of spectral densities can be used for finding the spectral characteristic and mean square error of the optimal linear estimator for functionals of stationary sequences in the extrapolation and interpolation problems for both cases of observations without and with a noise.

The expressions for the spectral characteristic and mean square error of the optimal estimator written in this way are convenient for finding the least favorable spectral densities and minimax spectral characteristics of estimators of the corresponding functionals in the extrapolation and interpolation problems for both cases of observations without and with a noise.

To solve the extrapolation problem for stationary sequences, Kolmogorov [8] (also see [18, 16, 17, 15]) used a method based on the factorization of the spectral density. This method is convenient for solving the extrapolation problems for observations without a noise, while the method of Theorem 3.1 fits the case of observations with a noise, as well.

Below we use the method based on the factorization of spectral densities and apply it to the estimation of a functional from observations without a noise.

Definition 3.1. Denote by $H_{\zeta}(n)$ the closed linear subspace of the Hilbert space $H$ generated by random variables $\zeta_{k j}, k \geq 1, j \leq n$. A sequence $\left\{\zeta_{j}, j \in \mathbb{Z}\right\}$ is called regular if $\bigcap_{n} H_{\zeta}(n)=\varnothing$.

A regular stationary sequence $\left\{\zeta_{j}, j \in \mathbb{Z}\right\}$ admits the canonical moving average representation for its components

$$
\zeta_{k j}=\sum_{u=-\infty}^{j} \sum_{m=1}^{M} d_{k m}(j-u) \varepsilon_{m}(u)
$$

(see [6]), where $\varepsilon_{m}(u), m=1, \ldots, M, u \in \mathbb{Z}$, are mutually orthogonal sequences with orthogonal values in $H$, that is

$$
\mathrm{E} \varepsilon_{m}(u) \overline{\varepsilon_{p}(v)}=\delta_{m p} \delta_{u v},
$$

where $M$ is the multiplicity of the stationary sequence $\left\{\zeta_{j}\right\}$, and where the sequences $d_{k m}(u), k=1,2, \ldots, m=1, \ldots, M, u=0,1, \ldots$, are such that

$$
\sum_{u=0}^{\infty} \sum_{k=1}^{\infty} \sum_{m=1}^{M}\left|d_{k m}(u)\right|^{2}=P_{\zeta}
$$


The optimal linear estimator of components of a regular stationary sequence $\left\{\zeta_{j}, j \in \mathbb{Z}\right\}$ constructed from observations of this sequence for $j<0$ can be written as follows:

$$
\hat{\zeta}_{k j}=\sum_{u=-\infty}^{-1} \sum_{m=1}^{M} d_{k m}(j-u) \varepsilon_{m}(u) .
$$

Since unknown values of components of a singular stationary sequence are estimated to be error free, one can restrict the consideration to the case of regular stationary sequences in the problem of optimal linear estimation.

The spectral density $f(\lambda)$ of a regular stationary sequence $\left\{\zeta_{j}, j \in \mathbb{Z}\right\}$ admits the canonical representation

$$
f(\lambda)=P(\lambda) P^{*}(\lambda), \quad P(\lambda)=\sum_{u=0}^{\infty} d(u) e^{-i u \lambda},
$$

where the matrix $d(u)=\left\{d_{k m}(u)\right\}_{k=1, \ldots, \infty}^{m=1, \ldots, M}$ is defined via the coefficients of canonical representation (18).

Using representations (18) and (19) for components of a regular stationary sequence $\left\{\zeta_{j}, j \in \mathbb{Z}\right\}$, one can write the mean square error of the optimal estimator $\hat{A} \zeta$ as follows:

$$
\begin{aligned}
\Delta(h(f) ; f) & =\mathrm{E}\left|\sum_{k=1}^{\infty} \sum_{j=0}^{\infty} a_{k j} \sum_{u=0}^{j} \sum_{m=1}^{M} d_{k m}(j-u) \varepsilon_{m}(u)\right|^{2} \\
& =\sum_{l=0}^{\infty} \sum_{m=1}^{M}\left|\sum_{k=1}^{\infty} \sum_{j=l}^{\infty} a_{k j} d_{k m}(j-l)\right|^{2}=\sum_{l=0}^{\infty}\left\|(\mathbf{A d})_{l}\right\|^{2}=\|\mathbf{A d}\|^{2},
\end{aligned}
$$

where $(\mathbf{A d})_{l}=\sum_{j=l}^{\infty} \vec{a}_{j}^{\top} d(j-l), l \geq 0$. If the second condition in (6) holds, then $\mathbf{A}$ is a compact operator.

Recall that the spectral characteristic $h(f)$ minimizes the mean square error

$$
\Delta(f)=\Delta(h(f) ; f)=\min _{h \in L_{2}^{-}(f)} \Delta(h ; f)=\|\mathbf{A d}\|^{2} .
$$

On the other hand, we derive from (9) that

$$
\Delta(h ; f)=\frac{1}{2 \pi} \int_{-\pi}^{\pi}\left[A\left(e^{i \lambda}\right)-h\left(e^{i \lambda}\right)\right]^{\top} f(\lambda) \overline{\left[A\left(e^{i \lambda}\right)-h\left(e^{i \lambda}\right)\right]} d \lambda .
$$

Thus it is natural to assume that

$$
h(f)=A\left(e^{i \lambda}\right)-X(\lambda),
$$

where $X(\lambda)$ is a vector column defined by

$$
\begin{aligned}
\sum_{l=0}^{\infty}\left\|(\mathbf{A d})_{l}\right\|^{2} & =\frac{1}{2 \pi} \int_{-\pi}^{\pi}\left(\sum_{l=0}^{\infty}(\mathbf{A d})_{l} e^{i l \lambda}\right)\left(\sum_{p=0}^{\infty}(\mathbf{A d})_{p} e^{i p \lambda}\right)^{*} d \lambda \\
& =\frac{1}{2 \pi} \int_{-\pi}^{\pi}\left(\sum_{l=0}^{\infty}(\mathbf{A d})_{l} e^{i l \lambda}\right) Q(\lambda) \underbrace{P(\lambda) P^{*}(\lambda)}_{f(\lambda)} Q^{*}(\lambda)\left(\sum_{p=0}^{\infty}(\mathbf{A d})_{p} e^{i p \lambda}\right)^{*} d \lambda \\
& =\frac{1}{2 \pi} \int_{-\pi}^{\pi} X^{\top}(\lambda) f(\lambda) \overline{X(\lambda)} d \lambda .
\end{aligned}
$$


Then $X(\lambda)=S\left(e^{i \lambda}\right) Q(\lambda)$, where

$$
S\left(e^{i \lambda}\right)=\sum_{l=0}^{\infty}(\mathbf{A d})_{l} e^{i l \lambda}
$$

and

$$
Q(\lambda)=\left\{q_{m k}(\lambda)\right\}_{m=1, \ldots, M}^{k=1, \ldots, \infty}
$$

is a matrix valued function satisfying the equation $Q(\lambda) P(\lambda)=I_{M}$. The spectral characteristic $h(f)$ is calculated according to

$$
h^{\top}(f)=A^{\top}\left(e^{i \lambda}\right)-S\left(e^{i \lambda}\right) Q(\lambda) .
$$

The above results are combined in the following assertion.

Theorem 3.2. Let $\{\zeta(t), t \in \mathbb{R}\}$ be a periodically correlated stochastic process such that the stationary sequence $\left\{\zeta_{j}, j \in \mathbb{Z}\right\}$ defined by relation (1) possesses the spectral density $f(\lambda)$ that satisfies the minimality condition (14).

Let the coefficients $\left\{\vec{a}_{j}, j=0,1, \ldots\right\}$ determining the functional $A \zeta$ in (5) satisfy conditions (6).

Then the spectral characteristic $h(f)$ and mean square error $\Delta(f)$ of the optimal linear estimator of the functional $A \zeta$ constructed from observations of the process $\zeta(t), t<0$, are calculated by relations (22) and (21). The optimal estimator $\hat{A} \zeta$ of the functional $A \zeta$ is calculated by (17).

Similarly to the reasoning above, one can prove the following assertion (that also follows from Theorem 3.2 ) for the functional:

$$
A_{N} \zeta=\int_{0}^{(N+1) T} a(t) \zeta(t) d t=\sum_{j=0}^{N} \vec{a}_{j}^{\top} \vec{\zeta}_{j} .
$$

Corollary 3.2. Let $\{\zeta(t), t \in \mathbb{R}\}$ be a periodically correlated stochastic process such that the stationary sequence $\left\{\zeta_{j}, j \in \mathbb{Z}\right\}$ constructed according to equality (1) possesses the spectral density $f(\lambda)$ that satisfies the minimality condition (14).

Then the spectral characteristic $h_{N}(f)$ and mean square error $\Delta_{N}(f)$ of the optimal estimator of the functional $A_{N} \zeta$ constructed from observations of the process $\zeta(t), t<0$, are calculated by

$$
\begin{gathered}
h_{N}^{\top}(f)=A_{N}^{\top}\left(e^{i \lambda}\right)-S_{N}\left(e^{i \lambda}\right) Q(\lambda), \\
\Delta_{N}(f)=\sum_{l=0}^{N}\left\|\left(\mathbf{A}_{\mathbf{N}} \mathbf{d}\right)_{l}\right\|^{2}=\left\|\mathbf{A}_{\mathbf{N}} \mathbf{d}\right\|^{2} \\
A_{N}\left(e^{i \lambda}\right)=\sum_{j=0}^{N} \vec{a}_{j} e^{i j \lambda}, \quad S_{N}\left(e^{i \lambda}\right)=\sum_{l=0}^{N}\left(\mathbf{A}_{\mathbf{N}} \mathbf{d}\right)_{l} e^{i l \lambda}, \quad\left(\mathbf{A}_{\mathbf{N}} \mathbf{d}\right)_{l}=\sum_{j=l}^{N} \vec{a}_{j}^{\top} d(j-l) .
\end{gathered}
$$

\section{Minimax (Robust) extrapolation}

One can use relations (11), (13), (15), (16), and (21)-(24) for evaluating the spectral characteristic and mean square error of the optimal estimator of the functional $A \zeta$ only if the spectral densities $f(\lambda)$ and $g(\lambda)$ of the stationary sequences $\left\{\zeta_{j}, j \in \mathbb{Z}\right\}$ and $\left\{\theta_{j}, j \in \mathbb{Z}\right\}$ constructed according to relations (11) and (2), respectively, are known. Otherwise, if the spectral densities are not known but a set $D=D_{f} \times D_{g}$ of admissible spectral densities is specified, then one can use the minimax approach to the problems of estimation of the functional depending on unknown values of the underlying process. We 
search for an estimator that minimizes the error simultaneously for all spectral densities belonging to a given class $D$.

Definition 4.1. Given a set of pairs of spectral densities $D=D_{f} \times D_{g}$, the spectral densities $f^{0}(\lambda) \in D_{f}$ and $g^{0}(\lambda) \in D_{g}$ are called the least favorable in $D$ for the optimal estimator of the functional $A \zeta$ if

$$
\Delta\left(f^{0}, g^{0}\right)=\Delta\left(h\left(f^{0}, g^{0}\right) ; f^{0}, g^{0}\right)=\max _{(f, g) \in D} \Delta(h(f, g) ; f, g) .
$$

Definition 4.2. Given a set of pairs of spectral densities $D=D_{f} \times D_{g}$, the spectral characteristic $h^{0}(\lambda)$ of the optimal estimator of the functional $A \zeta$ is called minimax (robust) if

$$
h^{0}(\lambda) \in H_{D}=\bigcap_{(f, g) \in D} L_{2}^{-}(f+g), \quad \min _{h \in H_{D}} \max _{(f, g) \in D} \Delta(h ; f, g)=\max _{(f, g) \in D} \Delta\left(h^{0} ; f, g\right) .
$$

Taking into account relations (11), (13), (15), (16), and (21)-(24), we prove the following auxiliary results.

Lemma 4.1. Spectral densities $f^{0}(\lambda) \in D_{f}$ and $g^{0}(\lambda) \in D_{g}$ satisfying condition (7) are the least favorable in the class $D$ for the optimal estimator of the functional $A \zeta$ if the Fourier coefficients of the functions

$$
\left(f^{0}(\lambda)+g^{0}(\lambda)\right)^{-1}, \quad f^{0}(\lambda)\left(f^{0}(\lambda)+g^{0}(\lambda)\right)^{-1}, \quad f^{0}(\lambda)\left(f^{0}(\lambda)+g^{0}(\lambda)\right)^{-1} g^{0}(\lambda)
$$

being the entries of the matrices $\mathbf{B}^{0}, \mathbf{D}^{0}$, and $\mathbf{R}^{0}$ determine a solution of the following optimization problem:

$$
\max _{(f, g) \in D}\left(\langle\mathbf{a}, \mathbf{R} \mathbf{a}\rangle+\left\langle(\mathbf{B})^{-1} \mathbf{D} \mathbf{a}, \mathbf{D a}\right\rangle\right)=\left\langle\mathbf{a}, \mathbf{R}^{0} \mathbf{a}\right\rangle+\left\langle\left(\mathbf{B}^{0}\right)^{-1} \mathbf{D}^{0} \mathbf{a}, \mathbf{D}^{0} \mathbf{a}\right\rangle .
$$

The minimax spectral characteristic $h^{0}=h\left(f^{0}, g^{0}\right)$ of the optimal estimator of the functional $A \zeta$ is calculated by equality (11) under the condition that $h\left(f^{0}, g^{0}\right) \in H_{D}$.

Lemma 4.2. A spectral density $f^{0}(\lambda) \in D_{f}$ satisfying condition (14) is the least favorable in the class $D_{f}$ for the optimal estimator of the functional $A \zeta$ constructed from observations of the process $\zeta(t), t<0$, if the Fourier coefficients of the function $\left(f^{0}(\lambda)\right)^{-1}$ constitute the matrix $\mathbf{B}^{0}$ that determine a solution of the following optimization problem:

$$
\max _{f \in D_{f}}\left\langle(\mathbf{B})^{-1} \mathbf{a}, \mathbf{a}\right\rangle=\left\langle\left(\mathbf{B}^{0}\right)^{-1} \mathbf{a}, \mathbf{a}\right\rangle \text {. }
$$

The minimax spectral characteristic $h^{0}=h\left(f^{0}\right)$ of the optimal estimator of the functional $A \zeta$ is calculated by equality (15) under the condition that $h\left(f^{0}\right) \in H_{D}$.

Lemma 4.3. A spectral density $f^{0}(\lambda) \in D_{f}$ satisfying condition (14) is the least $f a$ vorable in the class $D_{f}$ for the optimal estimator of the functional $A \zeta$ constructed from observations of the process $\zeta(t), t<0$, if it admits the canonical factorization

$$
f^{0}(\lambda)=\left(\sum_{u=0}^{\infty} d^{0}(u) e^{-i u \lambda}\right)\left(\sum_{u=0}^{\infty} d^{0}(u) e^{-i u \lambda}\right)^{*},
$$

where $\mathbf{d}^{\mathbf{0}}=\left\{d^{0}(u), u=0,1, \ldots\right\}$ is a solution of the following conditional extremum problem:

$$
\|\mathbf{A d}\|^{2} \rightarrow \max , \quad f(\lambda)=\left(\sum_{u=0}^{\infty} d(u) e^{-i u \lambda}\right)\left(\sum_{u=0}^{\infty} d(u) e^{-i u \lambda}\right)^{*} \in D .
$$

The minimax spectral characteristic $h^{0}=h\left(f^{0}\right)$ of the optimal estimator of the functional $A \zeta$ is calculated according to equality (22) under the condition that $h\left(f^{0}\right) \in H_{D}$. 
Lemma 4.4. A spectral density $f^{0}(\lambda) \in D_{f}$ satisfying condition (14) is the least favorable in the class $D_{f}$ for the optimal estimator of the functional $A_{N} \zeta$ constructed from observations of the process $\zeta(t), t<0$, if it admits the canonical factorization

$$
f^{0}(\lambda)=\left(\sum_{u=0}^{N} d^{0}(u) e^{-i u \lambda}\right)\left(\sum_{u=0}^{N} d^{0}(u) e^{-i u \lambda}\right)^{*}
$$

where $\mathbf{d}^{\mathbf{0}}=\left\{d^{0}(u), u=0,1, \ldots, N\right\}$ is a solution of the following conditional extremum problem:

$$
\left\|\mathbf{A}_{\mathbf{N}} \mathbf{d}\right\|^{2} \rightarrow \max , \quad f(\lambda)=\left(\sum_{u=0}^{N} d(u) e^{-i u \lambda}\right)\left(\sum_{u=0}^{N} d(u) e^{-i u \lambda}\right)^{*} \in D .
$$

The minimax spectral characteristic $h_{N}^{0}=h_{N}\left(f^{0}\right)$ of the optimal estimator of the functional $A \zeta$ is calculated according to equality (23) under the condition that $h_{N}\left(f^{0}\right) \in H_{D}$.

The proof of Lemmas 4.14 .4 is straightforward; they follow directly from Theorems 3.1 and 3.2 and their corollaries in view of the definition of the least favorable densities and minimax spectral characteristic.

The least favorable spectral densities $f^{0}(\lambda) \in D_{f}, g^{0}(\lambda) \in D_{g}$ and minimax spectral characteristic $h^{0}=h\left(f^{0}, g^{0}\right)$ form a saddle point of the function $\Delta(h ; f, g)$ in the set $H_{D} \times D$. The saddle point inequalities

$$
\Delta\left(h^{0} ; f, g\right) \leq \Delta\left(h^{0} ; f^{0}, g^{0}\right) \leq \Delta\left(h ; f^{0}, g^{0}\right), \quad \forall h \in H_{D}, \forall f \in D_{f}, \forall g \in D_{g},
$$

hold if $h^{0}=h\left(f^{0}, g^{0}\right), h\left(f^{0}, g^{0}\right) \in H_{D}$, and if $\left(f^{0}, g^{0}\right)$ is a solution of the following conditional extremum problem:

$$
\Delta\left(h\left(f^{0}, g^{0}\right) ; f, g\right) \rightarrow \sup , \quad(f, g) \in D,
$$

$$
\begin{aligned}
& \Delta\left(h\left(f^{0}, g^{0}\right) ; f, g\right) \\
& =\frac{1}{2 \pi} \int_{-\pi}^{\pi}\left[A^{\top}\left(e^{i \lambda}\right) g^{0}(\lambda)+\left(C^{0}\left(e^{i \lambda}\right)\right)^{\top}\right]\left(f^{0}(\lambda)+g^{0}(\lambda)\right)^{-1} \\
& \quad \times f(\lambda)\left(f^{0}(\lambda)+g^{0}(\lambda)\right)^{-1}\left[g^{0}(\lambda) \overline{A\left(e^{i \lambda}\right)}+\overline{C^{0}\left(e^{i \lambda}\right)}\right] d \lambda \\
& +\frac{1}{2 \pi} \int_{-\pi}^{\pi}\left[A^{\top}\left(e^{i \lambda}\right) f^{0}(\lambda)-\left(C^{0}\left(e^{i \lambda}\right)\right)^{\top}\right]\left(f^{0}(\lambda)+g^{0}(\lambda)\right)^{-1} \\
& \quad \times g(\lambda)\left(f^{0}(\lambda)+g^{0}(\lambda)\right)^{-1}\left[f^{0}(\lambda) \overline{A\left(e^{i \lambda}\right)}-\overline{C^{0}\left(e^{i \lambda}\right)}\right] d \lambda .
\end{aligned}
$$

The conditional extremum problem (31) is equivalent to the following unconditional extremum problem:

$$
\Delta_{D}(f, g)=-\Delta\left(h\left(f^{0}, g^{0}\right) ; f, g\right)+\delta((f, g) \mid D) \rightarrow \inf ,
$$

where $\delta((f, g) \mid D)$ denotes the indicator function of the set $D$. A solution $\left(f^{0}, g^{0}\right)$ of the latter problem is determined by the condition $0 \in \partial \Delta_{D}\left(f^{0}, g^{0}\right)$ (see [14]), which is a necessary and sufficient condition for the point $\left(f^{0}, g^{0}\right)$ to belong to the set of minimums of a convex function. Here $\partial \Delta_{D}\left(f^{0}, g^{0}\right)$ is a subdifferential of the convex functional $\Delta_{D}(f, g)$ at the point $(f, g)=\left(f^{0}, g^{0}\right)$.

Now we apply the conditions just obtained and find the least favorable spectral densities in some classes of admissible densities. 
5. Least favorable Spectral Densities in the Class $D_{0} \times D_{\varepsilon}$

Consider the problem of the minimax estimation of the functional $A \zeta$ from observations of the process $\zeta(t)+\theta(t), t<0$, under the condition that the spectral densities $f(\lambda)$ and $g(\lambda)$ of the stationary sequences $\left\{\zeta_{j}, j \in \mathbb{Z}\right\}$ and $\left\{\theta_{j}, j \in \mathbb{Z}\right\}$, respectively, constructed according to relations (11), (2), belong to the sets

$$
\begin{gathered}
D_{0}=\left\{f(\lambda) \mid \frac{1}{2 \pi} \int_{-\pi}^{\pi} \operatorname{Tr} f(\lambda) d \lambda=P_{\zeta}\right\}, \\
D_{\varepsilon}=\left\{g(\lambda) \mid \operatorname{Tr} g(\lambda)=(1-\varepsilon) \omega(\lambda)+\varepsilon u(\lambda), \frac{1}{2 \pi} \int_{-\pi}^{\pi} \operatorname{Tr} g(\lambda) d \lambda=P_{\theta}\right\},
\end{gathered}
$$

where $\omega(\lambda)$ is a known nonnegative function and $u(\lambda)$ is an unknown nonnegative function. The set $D_{0}$ is described by restrictions imposed on the moment of the spectral density $f(\lambda)$. The set $D_{\varepsilon}$ describes the model of a " $\varepsilon$-contamination" of a stochastic sequence $\left\{\theta_{j}, j \in \mathbb{Z}\right\}$.

To determine the pair of the least favorable densities $\left(f^{0}(\lambda), g^{0}(\lambda)\right)$ one can use the method of indefinite Lagrange multipliers. The Lagrange function for the conditional extremum problem (31) is given by

$$
\begin{aligned}
\mathcal{L}(f, g)= & -\Delta\left(h\left(f^{0}, g^{0}\right) ; f, g\right)+\alpha^{2}\left(\frac{1}{2 \pi} \int_{-\pi}^{\pi} \operatorname{Tr} f(\lambda) d \lambda-P_{\zeta}\right) \\
& +\beta^{2}\left(\frac{1}{2 \pi} \int_{-\pi}^{\pi} \operatorname{Tr} g(\lambda) d \lambda-P_{\theta}\right)+\gamma^{2}(\operatorname{Tr} g(\lambda)-(1-\varepsilon) \omega(\lambda)-\varepsilon u(\lambda)),
\end{aligned}
$$

where $\alpha^{2}, \beta^{2}$, and $\gamma^{2}$ are Lagrange multipliers. According to the Lagrange method, a solution $\left(f^{0}(\lambda), g^{0}(\lambda)\right)$ of the problem (31) satisfies the equation

$$
\begin{gathered}
\left(g^{0}(\lambda) \overline{A\left(e^{i \lambda}\right)}+\overline{C^{0}\left(e^{i \lambda}\right)}\right)\left(A^{\top}\left(e^{i \lambda}\right) g^{0}(\lambda)+\left(C^{0}\left(e^{i \lambda}\right)\right)^{\top}\right)=\alpha^{2}\left(f^{0}(\lambda)+g^{0}(\lambda)\right)^{2} \\
\left(f^{0}(\lambda) \overline{A\left(e^{i \lambda}\right)}-\overline{C^{0}\left(e^{i \lambda}\right)}\right)\left(A^{\top}\left(e^{i \lambda}\right) f^{0}(\lambda)-\left(C^{0}\left(e^{i \lambda}\right)\right)^{\top}\right) \\
=\left(\beta^{2}+\varphi(\lambda)\right)\left(f^{0}(\lambda)+g^{0}(\lambda)\right)^{2}
\end{gathered}
$$

where $\varphi(\lambda) \geq 0$ and $\varphi(\lambda)=0$ if $\operatorname{Tr} g^{0}(\lambda) \geq(1-\varepsilon) \omega(\lambda)$.

The latter equations (32) - (33) define the least favorable spectral densities in the class $D_{0} \times D_{\varepsilon}$.

The results above are combined in the following assertion.

Theorem 5.1. Let spectral densities $f(\lambda) \in D_{0}, g(\lambda) \in D_{\varepsilon}$ satisfy condition (7). Then the matrices of spectral densities $f^{0}(\lambda), g^{0}(\lambda)$ are the least favorable in the class $D_{0} \times D_{\varepsilon}$ for the optimal estimator of the functional $A \zeta$ if they satisfy relations (32), (33) and determine a solution of the extremum problem (25). The minimax spectral characteristic $h\left(f^{0}, g^{0}\right)$ of the optimal estimator of the functional $A \zeta$ is calculated by formula (11).

In the case of the estimation problem for the functional $A \zeta$ by observations without noise, Theorem 5.1 implies the following corollaries for the sets $D_{0}$ and $D_{\varepsilon}$ of admissible densities.

Corollary 5.1. Let a spectral density $f^{0}(\lambda) \in D_{0}$ satisfy condition (14). Then the matrix of spectral density $f^{0}(\lambda)$ is the least favorable in the class $D_{0}$ for the optimal estimator of the functional $A \zeta$ constructed from observations of the process $\zeta(t), t<0$, if it satisfies the relation

$$
\overline{C^{0}\left(e^{i \lambda}\right)}\left(C^{0}\left(e^{i \lambda}\right)\right)^{\top}=\alpha^{2}\left(f^{0}(\lambda)\right)^{2}
$$


and determines a solution of the extremum problem (26). The minimax spectral characteristic $h\left(f^{0}\right)$ of the optimal estimator of the functional $A \zeta$ is determined by relation (15).

Corollary 5.2. Let a spectral density $f^{0}(\lambda) \in D_{\varepsilon}$ satisfy condition (14). Then the matrix of the spectral density $f^{0}(\lambda)$ is the least favorable in the class $D_{\varepsilon}$ for the optimal estimator of the functional $A \zeta$ constructed from the observations of the process $\zeta(t), t<0$, if it satisfies the relation

$$
\overline{C^{0}\left(e^{i \lambda}\right)}\left(C^{0}\left(e^{i \lambda}\right)\right)^{\top}=\left(\beta^{2}+\varphi(\lambda)\right)\left(f^{0}(\lambda)\right)^{2}
$$

and determines a solution of the extremum problem (26). The minimax spectral characteristic $h\left(f^{0}\right)$ of the optimal estimator of the functional $A \zeta$ is determined by formula (15).

\section{Least favorable Densities in the Class $D_{0}$}

Consider the minimax estimation problem for the functionals $A \zeta$ and $A_{N} \zeta$ by using the observations of the process $\zeta(t), t<0$, for the set $D_{0}$ of spectral densities $f(\lambda)$ of stationary sequences $\left\{\zeta_{j}, j \in \mathbb{Z}\right\}$ constructed according to relation (11) and that admit the canonical factorization (20).

To determine the least favorable density $f^{0}(\lambda)$ we use the method of indefinite Lagrange multipliers. The Lagrange function for the conditional extremum function (28) is given by

$$
\begin{aligned}
\mathcal{L}(f)= & -\frac{1}{2 \pi} \int_{-\pi}^{\pi}\left(\sum_{l=0}^{\infty}(\mathbf{A d})_{l} e^{i l \lambda}\right) Q(\lambda) f(\lambda) Q^{*}(\lambda)\left(\sum_{l=0}^{\infty}(\mathbf{A d})_{l} e^{i l \lambda}\right)^{*} d \lambda \\
& +\alpha^{2}\left(\frac{1}{2 \pi} \int_{-\pi}^{\pi} \operatorname{Tr} f(\lambda) d \lambda-P_{\zeta}\right)
\end{aligned}
$$

where $\alpha^{2}$ is the Lagrange multiplier. Following the Lagrange method, we find that a solution $f^{0}(\lambda)$ of problem (28) satisfies the relation

$$
Q^{\top}(\lambda)\left(\sum_{l=0}^{\infty}(\mathbf{A d})_{l} e^{i l \lambda}\right)^{\top} \overline{\left(\sum_{l=0}^{\infty}(\mathbf{A d})_{l} e^{i l \lambda}\right) Q(\lambda)}=\alpha^{2} I_{\infty}
$$

which is equivalent to

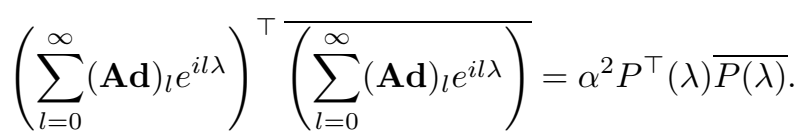

Removing the parentheses on the left hand side of (34) and then equating the coefficients of $e^{i l \lambda}, l \geq 0$, on both sides of (34) we get

$$
\sum_{t=0}^{\infty} d^{\top}(t) \sum_{p=0}^{\infty} \sum_{s=0}^{\infty} \vec{a}_{t+l+p} \vec{a}_{s+p}^{*} \overline{d(s)}=\alpha^{2} \sum_{t=0}^{\infty} d^{\top}(t) \overline{d(t+l)} .
$$

Given a fixed $t=0,1, \ldots$, we change the index of summation $t+l=r$ and apply the transposition in the left hand side of the latter equality. Then we obtain

$$
\sum_{p=0}^{\infty} \sum_{s=0}^{\infty} \overline{\vec{a}}_{r+p} \vec{a}_{s+p}^{\top} d(s)=\alpha^{2} d(r), \quad r=0,1, \ldots .
$$

If equalities (35) hold for all $r=0,1, \ldots$, then equality (34) holds, as well. 
The condition $f(\lambda) \in D_{0}$ yields the following restriction:

$$
\begin{gathered}
\frac{1}{2 \pi} \int_{-\pi}^{\pi} \operatorname{Tr}\left(\sum_{u=0}^{\infty} d(u) e^{-i u \lambda}\right)\left(\sum_{u=0}^{\infty} d(u) e^{-i u \lambda}\right)^{*} d \lambda=\sum_{u=0}^{\infty} \sum_{k=1}^{\infty} \sum_{m=1}^{M}\left|d_{k m}(u)\right|^{2} \\
=\sum_{u=0}^{\infty}\|d(u)\|^{2}=\|\mathbf{d}\|^{2}=P_{\zeta} .
\end{gathered}
$$

The results above allow one to state the following assertion.

Theorem 6.1. The spectral density (27) of the moving average sequence (18) is the least favorable in the class $D_{0}$ for the optimal estimator of the functional $A \zeta$. The sequence of matrices

$$
\mathbf{d}^{\mathbf{0}}=\left\{d^{0}(u), u=0,1, \ldots\right\}
$$

is defined by equality (35) and condition (36). The function $h\left(f^{0}\right)$ calculated according to formula (22) is the minimax spectral characteristic of the functional $A \zeta$.

Applying the Lagrange method of indefinite multipliers to the conditional optimization problem (30) and reasoning as above, one can prove for the functional $A_{N} \zeta$ that relation (34) in the case under consideration is given by

$$
\begin{aligned}
& \left(\sum_{l=0}^{N}\left(\mathbf{A}_{\mathbf{N}} \mathbf{d}\right)_{l} e^{i l \lambda}\right)^{\top} \overline{\left(\sum_{l=0}^{N}\left(\mathbf{A}_{\mathbf{N}} \mathbf{d}\right)_{l} e^{i l \lambda}\right)} \\
& =\alpha^{2}\left(\sum_{u=0}^{N} d^{0}(u) e^{-i u \lambda}\right)^{\top} \overline{\left(\sum_{u=0}^{N} d^{0}(u) e^{-i u \lambda}\right)} .
\end{aligned}
$$

Equality (37) follows from the equalities

$$
\sum_{p=0}^{N-r} \sum_{s=0}^{N-p} \overline{\vec{a}}_{r+p} \vec{a}_{s+p}^{\top} d(s)=\alpha^{2} d(r), \quad r=0,1, \ldots, N .
$$

Restriction (36) is rewritten as follows:

$$
\left\|\mathbf{d}_{\mathbf{N}}\right\|^{2}=\sum_{u=0}^{N}\|d(u)\|^{2}=\sum_{u=0}^{N} \sum_{k=1}^{\infty} \sum_{m=1}^{M}\left|d_{k m}(u)\right|^{2}=P_{\zeta} .
$$

The above results allow one to state the following assertion.

Theorem 6.2. The spectral density (29) of the moving average sequence

$$
\zeta_{k j}=\sum_{u=j-N}^{j} \sum_{m=1}^{M} g_{k m}(j-u) \varepsilon_{m}(u)
$$

is the least favorable in the class $D_{0}$ for the optimal estimator of the functional $A_{N} \zeta$. The sequence of matrices

$$
\mathbf{d}_{\mathbf{N}}^{\mathbf{0}}=\left\{d^{0}(u), u=0,1, \ldots, N\right\}
$$

is determined by equalities (38) and condition (39). The function $h_{N}\left(f^{0}\right)$ calculated by formula (23) is the minimax spectral characteristic of the functional $A_{N} \zeta$. 


\section{Concluding Remarks}

A method is proposed for solving the optimal linear estimation problem for functionals depending on unknown values of periodically correlated stochastic processes. The method is based on reducing the problem to a corresponding problem for stationary (infinite dimensional) sequences. We also use the property that the mean square error can be written as a linear functional in the space $L_{1} \times L_{1}$ with respect to $(f, g)$, which allows one to solve the conditional extremum problem and find a minimax estimator.

There are a number of papers devoted to periodically correlated sequences, and only a few of them deal with periodically correlated processes (see, for example, the papers by Makagon [10] and [11]).

In the current paper, we establish a formula for the mean square error and for the spectral characteristic in the optimal estimation problem for the functional

$$
A \zeta=\int_{0}^{\infty} a(t) \zeta(t) d t
$$

that depends on unknown values of a periodically correlated stochastic process $\zeta(t)$. The estimator is constructed from observations of the process

$$
\zeta(t)+\theta(t), \quad t<0,
$$

where $\theta(t)$ is a periodically correlated stochastic process being uncorrelated with $\zeta(t)$. The problem is considered for both cases of observations with and without a noise. We show that the periodically correlated stochastic process generating the stationary (infinite dimensional) one-sided moving average sequences is the least favorable for the optimal linear estimation of the functional $A \zeta$ in the class of admissible spectral densities with restrictions imposed on the moments.

\section{BIBLIOGRAPHY}

1. E. G. Gladyshev, Periodically and semi-periodically correlated random processes with continuous time, Teor. Veroyatnost. Primenen. 8 (1963), no. 2, 184-189; English transl. in Theory Probab. Appl. 8 (1963), 173-177. MR0152005 (27:1986)

2. U. Grenander, A prediction problem in game theory, Ark. Mat. 3 (1957), 371-379. MR0090486 $(19: 822 \mathrm{~g})$

3. I. Dubovets'ka, Extrapolation problem for a functional of a periodically correlated sequence, Visnyk Kyiv University. Mat. Mekh. 25 (2011), 22-26. (Ukrainian)

4. I. I. Dubovets'ka and M. P. Moklyachuk, Filtration of linear functionals of periodically correlated sequences, Teor. Imovir. Mat. Stat. 86 (2012), 43-55; English. transl. in Theory Probab. Math. Statist. 86 (2013), 51-64. MR2986449

5. I. I. Dubovets'ka, O. Yu. Masyutka, and M. P. Moklyachuk, Interpolation of periodically correlated stochastic sequences, Teor. Imovir. Mat. Stat. 84 (2011), 43-56; English transl. in Theory Probab. Math. Statist. 84 (2012), 43-56. MR2857415 (2012k:60099)

6. G. Kallianpur and V. Mandrekar, Spectral theory of stationary H-valued processes, J. Multivariate Anal. 1 (1971), 1-16. MR0301788 (46:943)

7. S. A. Kassam and H. V. Poor, Robust techniques for signal processing: A survey, Proc. IEEE. 73 (1985), 433-481.

8. A. N. Kolmogorov, Probability theory and mathematical statistics, Selected works, "Nauka", Moscow, 1986; English transl., with a preface by P. S. Aleksandrov, translated from the Russian by G. Lindquist, translation edited by A. N. Shiryayev, Mathematics and its Applications (Soviet Series), vol. 26, Kluwer Academic Publishers Group, Dordrecht, 1992. MR.1153022 (92j:01071)

9. O. M. Kurkin, Yu. B. Korobochkin, and S. A. Shatalov, Minimax Processing of Information, "Energoatomizdat", Moscow, 1990. (Russian) MR1060955 (91j:93102)

10. A. Makagon, Induced stationary process and structure of locally square integrable periodically correlated processes, Studia Math. 136 (1999), no. 1, 71-86. MR1706587 (2000i:60035)

11. A. Makagon, Characterization of the spectra of periodically correlated processes, J. Multivariate Anal. 78 (2001), 1-10. MR1856263 (2002i:60076) 
12. M. P. Moklyachuk, Robust Estimates for Functionals of Stochastic Processes, "Kyivs'kyi Universytet", Kyiv, 2008. (Ukrainian)

13. M. P. Moklyachuk and A. Yu. Masyutka, Minimax-Robust Estimation Technique for Stationary Stochastic Processes, LAP Lambert Academic Publishing, 2012.

14. M. P. Moklyachuk, Nonsmooth Analysis and Optimization, "Kyivs'kyi Universytet", Kyiv, 2008. (Ukrainian)

15. Yu. A. Rozanov, Stationary Stochastic Processes, 2nd ed., Teoriya Veroyatnostei i Matematicheskaya Statistika [Probability Theory and Mathematical Statistics], vol. 42, "Nauka", Moscow, 1990. (Russian) MR1090826 (92d:60046)

16. A. M. Yaglom, Correlation theory of stationary and related random functions. Vol. 1: Basic results, Springer Series in Statistics, Springer-Verlag, New York, 1987. MR0893393 (89a:60105)

17. A. M. Yaglom, Correlation theory of stationary and related random functions. Vol. 2: Supplementary notes and references, Springer Series in Statistics, Springer-Verlag, New York, 1987. MR915557(89a:60106)

18. Norbert Wiener, Extrapolation, Interpolation and Smoothing of Stationary Time Series. With Engineering Applications, The Technology Press of the Massachusetts Institute of Technology, Massachusetts Institute of Technology, Cambridge, Mass., 1949. MR.0031213 (11:118j)

Department of Probability Theory, Statistics, and Actuarial Mathematics, Kyiv National Taras Shevchenko University, Volodymyrs'Ka Street, 64, Kyiv 01601, Ukraine

E-mail address: idubovetska@gmail.com

E-mail address: mmp@univ.kiev.ua

Received 04/OCT/2012

Translated by V. SEMENOV 